\title{
Candidate solutions to improve Wireless Mesh Networks WMNs performance to meet the needs of Smart Grid applications - Survey paper
}

\author{
Eman A Arabyat \\ Department of Electrical and Computer Engineering, North Carolina State University, \\ Raleigh, NC, USA \\ eaarabya@ncsu.edu
}

\begin{abstract}
96\% market share of existing Smart Grid network installations is wireless mesh networks [1]. The paper starts by justifying the selection of WMNs as opposed to any other communication technology based on quantifying the bandwidth/latency/QoS constraints of a number of Smart Grid applications. The main objective of this paper, however, is to discuss some optimization techniques that found in the literature and can be implemented to overcome some of the challenges currently being faced by WMNs deployment in Smart Grid's NANs. Hybrid WMN (HWMN) is proposed as an optimization on the topology level to leverage WMNs convergence. Distributed Autonomous Data Routing DADR, multigate and diversity routing are optimizations on the protocol level to minimize the down time of WMNs. Cognitive Radio is investigated as an optimization on the physical level. The paper also explores the feasibility of using Wireless Software Defined Networks WSDN to improve the overall visibility and manageability of WMNs.
\end{abstract}

\section{Keywords}

Smart Grid, Wireless Mesh Networks, Home Area Network, Neighbor area networks, ZigBee, QoS, Hybrid wireless mesh network, Cognitive Radio, wireless software defined network, OpenFlow.

\section{Introduction}

According to the Energy Independence and Security Act of 2007, smart grid communication networks should provide secure and reliable end-to-end two-way communications [2].

Hence the success of the smart grid depends on a successful communication between the smart meters and an advanced metering infrastructure (AMI) heads. Smart Grid has many applications each with specific bandwidth, latency and QoS constraints that should be taken into consideration when selecting one communication technology in favor of another.

The first section of the paper maps the Smart Grid applications to its requirements. The second \& third sections deal with Smart Grid communication Infrastructure, WMNs in particular. Section four discusses the major challenges faced by WMNs .Section five proposes the potential of deploying hybrid wireless mesh network with customized protocols to improve its performance, section six discusses the use of cognitive radio as a work around solution of the scarcity of free channels in the $2.4 \mathrm{GHz}$ band. Section eight proposes the use of wireless software defined 
networks WSDN as an approach to provide better manageability over the communication system of smart grid.

\section{Smart Grid requirement \& applications}

When talking about smart grid it's important to understand that it's not a single discipline technology, it's rather a multidisciplinary technology that requires collaborative efforts from power, mechanical, civil engineering, communication and information disciplines and many others to pave the way for a successful smart grid existence.

Smart Grid promises many applications, but time of use pricing has generated the biggest hype. For this application to manifest we need to make the appliances "talk"! Hence sensors are used to send raw information about their consumption to the energy utilities which provide the corresponding price charge through an on-line web service [3]. For example, the customer might exploit any price reduction offered by the utility due to a peak in energy production of any given renewable resource. This creates a win-win situation for both the customer and the utility (because the energy won't get wasted).

This very application brings the notion of "the Internet of Everything (IoE)" which implies that all the devices (objects) can be controlled, sensed and managed through a corresponding IP. Due to the scarcity in IPV4 addresses ZigBee-enabled home appliances scattered around the home need to be aggregated in a smart meter that acts as a proxy to connect to Home Area Network (HAN), at least until IPV6 is here!

The requirements for "time of use pricing" application aren't very strict in terms of latency; it can tolerate few seconds of delay without major effects. However, in terms of bandwidth, given the fact that there are an estimated sixty-five million smart meters planned for deployment by 2020 [4], hundreds of megabits are needed!

Integrating renewable energy sources (like wind G1 in the below figure) in the electric grid in form of Static Var Compensator (SVC) micro grid is another important application of the smart grid. Many sensors are involved in sensing power flow magnitudes and directions in distribution lines, power flow from the wind generator, bus voltages and line currents. All these sensors need to communicate with the utility to enable real time response from the utility.

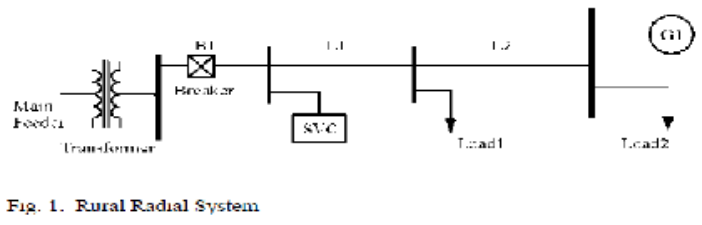

Figure 1: Rural Radial System [5]

According to the research done in [5] Estimated both bandwidth and latency requirements for this application; the minimum bandwidth for each smart meter placed in this system is 2-5 Mbps without even considering the routing protocol overheads. A maximum tolerance of 6 cycles, or $100 \mathrm{~ms}$ is estimated as latency bound.

Another two applications that require both real-time communication and high bandwidth are outage data notification to utilities and remote service disconnection of unnecessary loads during peak demands periods. In case of outage, many packets might be generated in a short amount of 
International Journal of Computer Science, Engineering and Information Technology (IJCSEIT), Vol.3,No.4,August 2013

time which might create congestions and bottlenecks. This can be avoided if constraints like high bandwidth and differentiated QoS of outage messages are considered in the network design.

The strict latency and high bandwidth requirements should serve as a justification for using one type of communication in favor of another.

\section{Smart Grid communication infrastructure}

The infrastructure of Smart Grid's two-way communication consists of three layers; Home Area Networks (HAN), Neighbor area networks (NAN) and wide area networks (WAN) [2, 6].

HAN consists of a group of sensors connected to a smart meter. The metering module (MM) inside the smart meter records the consumption of the energy for a given home and the meter controlling system (MCS) transmits the collected information. The chosen network standard in HAN is 802.15.4 Zigbee [6].

NAN is formed by connecting multiple MCSs of HANs that are geographically close. It can be deployed by a number of diverse communications technologies that fall in two categories; wired and wireless.

Broadband over Power Lines (BPL) is an example on wired networks. Cellular, WiMAX, and wireless mesh network (WMN) are examples on wireless networks, all four communications technologies are currently being tested for smart grid's NAN [1].

BPL deployment is challenged by a number of problems; the most important one is that BPL depends on the electrical grid itself, this means if a blackout happens, the needed communication will no longer exists! Another challenge faces BPL deployment is the fact that power lines are inherently noisy; power line communication (PLC) signals can't overcome this easily.

Cellular doesn't suffer from the problems BPL suffers from but one of the problems reference [1] suggests that it might affect cellular network as a choice for smart grid NAN communication is the fact that deployment of cellular might simplify the accessibility to the grid for terrorists or others who seeks to disrupt service delivery or create blackouts. However, this is not a vulnerability of cellular networks in particular but in fact to all wireless technologies considered for smart grid!

As a matter of fact, Cellular is being adopted currently from certain vendors in smart grid; Echelon's Networked Energy Services (NES) system has chosen T-Mobile's Global System for Mobile Communications (GSM) for its smart grid deployment. A SIM is inserted in each Echelon's meter to enable the communication between smart meters and the back-haul utilities [7]. Although cellular systems may reduce the CPEX, utilities need to spend a costly licensed spectrum fees to build the infrastructure. Cellular also suffers from other problems like poor coverage in rural areas as well as bandwidth limitation (hundreds of Kilobits) in GPRS, CDMA or 3G. LTE, however, might become a good enabler for IoEs or Smart Grid [8].

WiMAX in NAN is still fighting over market acceptance. "I don't think the future is very promising for WiMax" said Anssi Vanjoki, Nokia's head of sales and manufacturing [1]. However WiMax can be used in WAN's smart grid deployments because it can provide communication at a distance as long as 25 miles [6]. 
International Journal of Computer Science, Engineering and Information Technology (IJCSEIT), Vol.3,No.4,August 2013

The most important technology among the above, which by far the most deployed in the US Smart Grid And holds 96\% market share of existing smart grid network installations [1] is wireless mesh networks. WMNs provide 54-300 Mbps of bandwidth, they operate in the unlicensed band of $2.4 \mathrm{GHz}$ or $900 \mathrm{MHz}$. WMNs are self-organizing; require no manual address/route/channel assignments because WMN routing mechanisms provide redundant paths between the sender and receiver of wireless connections which give some guarantees for reliability [9].

\section{Wireless Mesh Networks deployment in Smart Grid}

WMNs are deployed in two ways in today's smart grid; Client WMNs: where each smart meter acts as both a meter and an access point for other meters at the same time [10].

PG\&E Company equips every smart device with a radio module and each of them routes the metering data through nearby meters [7] until it reaches the backhaul access point.

The other deployment is Infrastructure/Backbone WMNs (IWMN) where the meter acts as a simple relay to forward the packet toward an access point that handles the routing. This deployment is adopted by SkyPilot Company. Since a simpler meter is implemented a reduction of the overall cost of the system might be achieved [7].

\section{Wireless Mesh Networks challenges in Smart Grid}

Any of the two deployments in the smart grid has its pros and cons. While the second scenario can reduce the cost of overall system since a simpler meter is implemented, it can result in lowering the bandwidth if an optimum node density wasn't taken into consideration.

Both deployments also suffers from reliability issues, because the state of the link may differ rapidly between the time when the route was discovered, and the time when the data is forwarded; one second the wireless access point has a Line Of Sight, the other second a van arrives and block this LOS. Unreliable wireless links can cause frequent link and route failures, creating a major challenge for routing protocols to constantly repair routes and find alternate paths in a timely manner to meet the low latency demand of smart grid applications [11].

Another challenge faced by WMNs is the fact that the license-free $2.4 \mathrm{GHz}$ ISM band is extremely crowded by many devices operating in this range. This leads to interference problems that cause some packets to be dropped or delayed.

The projected increase in smart meters number imposes a manageability challenge on WMNs that has to be concurred for the success of smart grid.

Thus, given the smart grid application requirements, novel topologies and customized protocols should be considered to mitigate the scalability, manageability, and reliability and interference challenges faced by the WMN implementation in smart grid.

Security is also an issue in WMNs in smart grid but it's beyond the scope of this paper.

The coming sections of the papers will discuss some solutions from the literature to overcome the WMNs challenges in smart grid deployment. Some of the solutions are; enhancing the routing protocols to solve reliability and scalability issues. Using Cognitive radios to solve interference 
International Journal of Computer Science, Engineering and Information Technology (IJCSEIT), Vol.3,No.4,August 2013

challenges. A speculation on deploying Wireless SDN networks to solve the manageability problem in smart grid is proposed as well.

\section{Improving reliability \& scalability}

Research done in both $[12,13]$ Suggests the use of hybrid wireless mesh network (HWMN) to improve connectivity and convergence in WMNs. HWMN is a combination of infrastructure and client mesh network. Mesh clients (which in the smart grid are the smart meters) can access the network through mesh routers (access points) as well as directly meshing with other mesh clients (meters). The use of HWMNs might solve the convergence and fast recovery on the expense of throughput; the system might suffer now from three bottlenecks instead of two! One at the backhaul smart meter, the other at the access point and the last will be at the backhaul access point. [14] Shows that the throughput of each node decreases as a function of $O(1 / n)$ where $n$ is the number of mesh nodes. Hence if HWMN is to be followed, a topology planning tool should be used to measure the best oversubscription rate of the number of smart meters that should be served by one access point, and the number of access points that should be served by a backhaul AP. The tool should consider other factors as well, like channel assignments schemes, routing protocols, distances between points, etc.[15]

\section{Using Cognitive Radio to mitigate interference in Smart Grid}

One integrated strategy to achieve high degree of reliability, self-configuring and self-healing in HWMNs is proposed in [2] involves three components; multigate routing, real-time traffic scheduling, and multichannel (MC) aided wireless mesh routing.

Multigate reserve path routing suggests that every smart meter will have the routing flexibility to select one gateway out of many neighbour gateway access points based on the best path each access point offers. The other paths will be used in case of a link failure. While redundant paths are considered a desired feature, flooding the network with broadcast packets is undesirable especially in smart grid deployment, thus a Distributed Autonomous Depth-First Routing (DADR) proposed in reference [11] can be used instead. The research done in [2 ] claims that multigate routing with real-time back pressure packet scheduling will improve the network performance. The backpressure algorithm scheme works as this; every node evaluates the route to the next hop neighboring node based on the queue size and best path metric (BPM) which can be path quality metric, such as the Air-time link quality, or hop count. The Next Hop Selection (NHS) is selected based on the minimum congested path, and this evaluation process happens at every hop up to the backhaul wireless access point. This leads to an end to end best path selection. In the event of link failure, the neighbour with the second lowest BM value is used to construct a new path [2].

One major limitation on this approach, that it's being centralized rather than decentralized, so the approach proposed here is a heuristic one. However, it can be implemented if wireless software defined were used-will be explained in next sections.

Another solution proposed in [16] to take advantage of path diversity is to utilize both links instead of keeping one as standby, one of the links will be used to send data, and the other will be used to send meta data. 


\section{Using Cognitive Radio to mitigate interference in smart grid}

Smart grid devices need to avoid interference as much as possible to provide high data rates of up to tens of Mbps. Agile spectrum access enabled by Cognitive Radio CR technology can be adopted by the smart grid to make this happen. CR exploits the underutilized TV white spaces TVWS (guard bands) which have been recently permitted to be used in wireless communication by the federal communications commission (FCC). TV bands are ideal for long distance mass data transmission which meets the smart grid applications' demands.

A scenario to implement cognitive radio in client WMN NAN is given in [17]. The backhaul access point and smart meters are equipped with $\mathrm{CR}$ and utilize unused spectrum dynamically to communicate with each other. The backhaul access point gets to know the unutilized channels from a database setting somewhere in the utility.

An Infrastructure WMN scenario to implement cognitive radio in NAN is given in [18]. The smart meter communicates with its gateway access point in licensed band using cognitive radio technology to save the cost of buying spectrum bands. The gateway access point manages the access of smart meters and distributes spectrum bands to them according to their transmission demands. The unlicensed band is still needed as the availability of white spaces shouldn't be taken for granted; if the licensed spectrum band is needed by primary user at any time the secondary users of the band should migrate to the unlicensed band again.

Cognitive Radio process indicated in research done in Reference [19] is shown below,

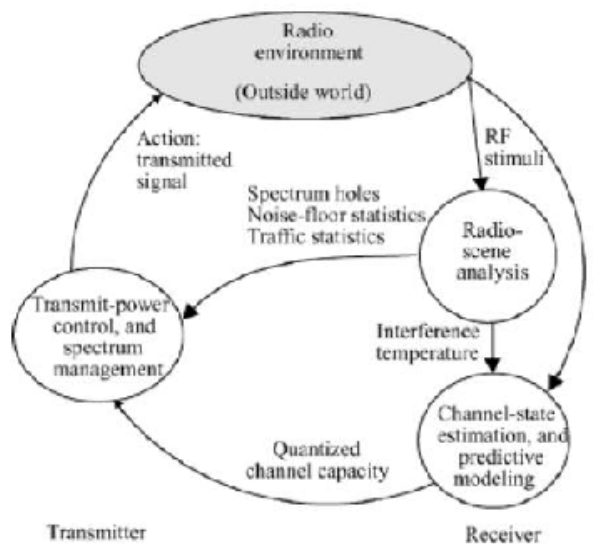

Figure 2: Cognitive Radio Process [16]

The receiver starts by sensing the medium to estimate the interference and detect if there are any underutilized bands the second step is to predict the available bandwidth to be used and the last step is to transmit power control and spectrum management signals.

The use of cognitive radio might be very promising for smart grid applications. However, it requires devices to have high-quality spectrum-sensing and efficient algorithms for exchanging spectrum-sensing data between nodes. 
International Journal of Computer Science, Engineering and Information Technology (IJCSEIT), Vol.3,No.4,August 2013

\section{Using Software Defined Network in Wireless Mesh Networks for better manageability \& less delay}

Not enough literature has been found regard using wireless software defined network in Smart Grid. However, the literature that was found about using the WSDN in wireless mesh networks can be used to infer some conclusions about the feasibility of using WSDN in smart grid.

WSDN relies basically on decoupling the control plane from the data plan. WSDN consists of three components, a centralized control server that runs Network Operating System (NOX) which takes care of setting the forwarding tables. The second component is simple access points with flow tables containing the set of rules for flow-processing, as well as a small monitoring and control agent to allow the NOX to query the APs to provide monitoring information about link quality, channel utilization etc...OpenFlow protocol is the third component of WSDN which tunnels the information from the data plane of APs to the NOX.

In Section five the backpressure algorithm mentioned was a heuristic approach, however wireless software defined network discussed in [20] suggests that backpressure routing or flow based routing might be feasible with the help of OpenFlow protocol. Flow based routing is extremely beneficial for real-time applications of smart grid because the amount of traffic in the network is considered before making routing decisions, thus it produces the minimum average delay. However in order for OpenFlow to succeed in WMNs it needs to be able to react to changes in the network in a rapid manner. The overhead of control messages should be kept to minimum as well.

The second problem can be solved by dual radio access points. It can dedicate one radio for control and the other for traffic, or by using two SSIDs each will serve as a dedicated VLAN.

Evaluation of general throughput of wireless mesh network that implements OpenFlow-wither it has to carry simple rules or complex rules, as opposed to the one that doesn't is given in [20] using a test bed of three nodes is shown below

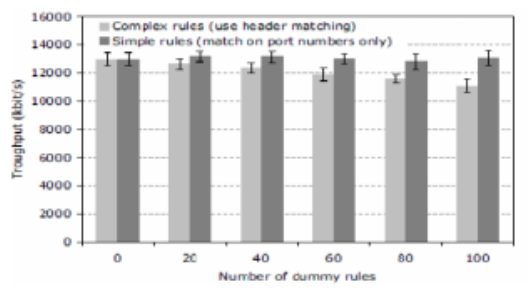

Figure 3: effect of rules on the throughput [20]

As shown in Figure 3, when 100 complex (MAC and IP addresses) rules need to be processed for each packet a $15 \%$ degradation of performance is exhibited. Less number of simple rules has less impact on performance but it is still less than the $20 \mathrm{Mbps}$ achieved without OpenFlowmentioned in [20]. fortunately, Smart Grid doesn't need much rules since nodes are stationary as opposed to mobile nodes, plus if the rules were tuned carefully Openflow's small degradation of the performance will be a fair price to be paid to select the less congested path and give better manageability over the system.

The number of rules also reflects on the size of control traffic that is propagated in the wireless mesh network. The difference between OSLR's control traffic as opposed to Open flow is shown in Figure 4 


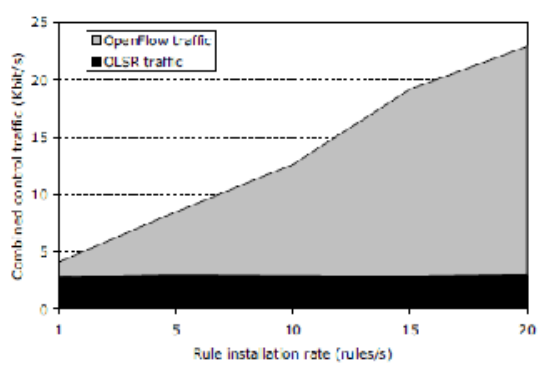

Figure 4: Total control traffic caused by OSLR and OpenFlow [20]

As shown in Figure 4, while OLSR traffic stays constant, OpenFlow's control traffic increases as the rule installation rate increases. But fortunately again, the number of rules in case of load balancing (which is what concerns us in smart grid) might be less than the number of rules in case of mobility and handover. Moreover, the number of heart beat signals (link quality, channel utilization) might be reduced if multiple nodes combine their signals in one packet!

\section{Conclusion}

Some of Smart Grid applications are imposing strict requirements on WMNs. While the use of HWMN is advantageous for many reasons, it's not sufficient to improve the performance and the scalability of Smart Grid WMNs, it needs to be combined with optimization techniques on the protocols level, the physical level and the manageability level. The paper proposed some protocols from the literature like Multigate reserve path routing to optimize the reliability and convergence and (DADR) protocol to be used instead of broadcast. Cognitive Radio might help in avoiding the crowded band of $2.4 \mathrm{GHz}$ but it requires the devices to have high-quality spectrumsensing and efficient algorithms for exchanging spectrum-sensing signals. The use of wireless software defined in Smart Grid was explored at the last part. A conclusion has been drawn that with the help of dual radio access points and the proper tuning for forwarding rules, WSDN can lead to a better managed Smart Grid communication infrastructure on the expense of noticeable degradation in throughput

\section{References}

[1] ConCentris Systems, MakaMesh. The Case for Using Wireless Mesh Technology in Smart Grid Communication Networks [Online]. Available: http://www.concentrissystems.com/downloads/SmartGridWMAdvantages.pdf

[2] Gharavi, H.; Bin Hu, "Multigate Communication Network for Smart Grid," Proceedings of the IEEE, vol.99, no.6, pp.1028,1045,„June2011 doi: 10.1109/JPROC.2011.2123851

[3] Aravinthan,V; Karimi, B; Namboodiri, V; Jewell,W; "Wireless Communication for Smart Grid Applications at Distribution Level - Feasibility and Requirements"

[4] EDX Wireless. Technology white papers, smart planning for Smart Grid AMI mesh networks [Online]. Available: http://www.edx.com/resources/documents/EDX_WP_Smart_Grid_AMI_Mesh_Networks_May_11.p df

[5] Sood, V.K.; Fischer, D.; Eklund, J.M.; Brown, T., "Developing a communication infrastructure for the Smart Grid," Electrical Power \& Energy Conference (EPEC), 2009 IEEE , vol., no., pp.1,7, 22-23 Oct. 2009 doi: 10.1109/EPEC.2009.5420809 
International Journal of Computer Science, Engineering and Information Technology (IJCSEIT), Vol.3,No.4,August 2013

[6] Yichi Zhang; Weiqing Sun; Lingfeng Wang; Hong Wang; Green, Robert C.; Alam, Mansoor, "A Multi-Level Communication Architecture of Smart Grid Based on Congestion Aware Wireless Mesh Network" , North American Power Symposium 2009 -NAPS , 2011, DOI: 10.1109/NAPS.2011.6025169

[7] Gungor, V.C.; Sahin, D.; Kocak, T.; Ergut, S.; Buccella, C.; Cecati, C.; Hancke, G.P., "Smart Grid Technologies: Communication Technologies and Standards," Industrial Informatics, IEEE Transactions on , vol.7, no.4, pp.529,539, Nov.2011 doi: 10.1109/TII.2011.2166794

[8] A.Clark,C.Pavlovski, Wireless networks for the smart energy grid: application aware networks ,in: Proceedings of the International MultiConference of Engineers and Computer Scientists, vol.2,2010.

[9] Kim, Jaebeom; Kim, Dabin; Lim, Keun-Woo; Ko, Young-Bae; Lee, Sang-Youm, "Improving the reliability of IEEE 802.11 s based wireless mesh networks for smart grid systems," Communications and Networks, Journal of , vol.14, no.6, pp.629,639, Dec. 2012 doi: 10.1109/JCN.2012.00029

[10] D. J. Leeds. (2012,Jan.16). Who Are the Top Ten Vendors in Smart Grid? [Online]. Available http://www.greentechmedia.com/articles/read/who-are-the-top-ten-vendors-in-smart-grid

[11] Iwao, T.; Yamada, K.; Yura, M.; Nakaya, Y.; Cardenas, A.A.; Sung Lee; Masuoka, R., "Dynamic Data Forwarding in Wireless Mesh Networks," Smart Grid Communications (SmartGridComm), 2010 First IEEE International Conference on , vol., no., pp.385,390, 4-6 Oct. 2010 doi: 10.1109/SMARTGRID.2010.5622074

[12] Akyildiz, I.F.; Xudong Wang, "A survey on wireless mesh networks," Communications Magazine, IEEE , vol.43, no.9, pp.S23,S30, Sept. 2005 doi: 10.1109/MCOM.2005.1509968

[13] Benyuan Liu; Zhen Liu; Towsley, D., "On the capacity of hybrid wireless networks," INFOCOM 2003. Twenty-Second Annual Joint Conference of the IEEE Computer and Communications. IEEE Societies , vol.2, no., pp.1543,1552 vol.2, 30 March-3 April 2003 doi: 10.1109/INFCOM.2003.1208989

[14] Jangeun Jun; Sichitiu, M.L., "The nominal capacity of wireless mesh networks," Wireless Communications, IEEE, vol.10, no.5, pp.8,14, Oct 2003 doi: 10.1109/MWC.2003.1241089

[15] Micronet Communications, Inc. (2011). Smart Grid Spectrum Development \& Planning Tool [Online]. http://www.micronetcom.com/assets/Smart\%20Grid\%20Spectrum\%20Development\%20and\% OPlanning\%20Tool.pdf

[16] Resilience Against Single Attacks in Wireless Mesh Networking for the Smart Grid

[17] Jianfeng Wang; Ghosh, M.; Challapali, K., "Emerging cognitive radio applications: A survey," Communications Magazine, IEEE , vol.49, no.3, pp.74,81, March 2011 doi: 10.1109/MCOM.2011.5723803

[18] Rong Yu; Yan Zhang; Gjessing, S.; Chau Yuen; Shengli Xie; Guizani, M., "Cognitive radio based hierarchical communications infrastructure for smart grid," Network, IEEE , vol.25, no.5, pp.6,14, September-October 2011 doi: 10.1109/MNET.2011.6033030

[19] Dehalwar,V, Kolhe,M,*, Kolhe,S; "Cognitive Radio Application for Smart Grid" International Journal of Smart Grid and Clean Energy, vol. 1, no. 1, September 2012

[20] Dely, P.; Kassler, A.; Bayer, N., "OpenFlow for Wireless Mesh Networks," Computer Communications and Networks (ICCCN), 2011 Proceedings of 20th International Conference on , vol., no., pp.1,6, July 31 2011-Aug.4.2011 doi: 10.1109/ICCCN.2011.6006100

\section{Eman Arabyat}

Is a Fulbright scholar currently doing her MS in Computer Networking at North Carolina State University. She performs her research in wireless networking and the application of wireless technologies for the Smart Grid. She is interning at IBM in Storage Area Networks department. Her bachelor's degree is in Computer Engineering that she received in 2010.

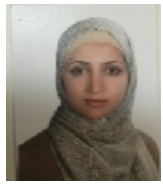

